\title{
IXODES PSEUDOHOLOCYCLUS Senevet, 1968, EST UN SYNONYME DE IXODES PETAURIST AE Warburton 1933
}

\author{
par G. SENEVET \\ Laboratoire de Parasitologie et de Mycologie ( $\mathrm{P}^{\mathrm{r}} \mathrm{M}$. LARIVIÈRE) \\ U.E.R. des Cordeliers, 15, rue de l'Ecole-de-Médecine, F 75 -Paris $\left(6^{\circ}\right)$
}

J'ai décrit, en 1968, une nymphe trouvée dans les collections du Laboratoire de Parasitologie de la Faculté de Médecine de Paris sous l'étiquette «Ixodes holocyclus».

Ixodes holocyclus étant une espèce australienne, dont la nymphe est très différente de celle de Paris, cette dernière ne pouvait pas être rapportée à l'espèce holocyclus.

Ayant cherché dans les descriptions de Sharif (1928) s'il n'existait pas, dans l'Inde, une espèce dont la nymphe correspondrait à celle de Paris, je n'en ai pas trouvé et j'ai considéré comme nouvelle, sous le nom de pseudoholocyclus, la nymphe que je décrivais.

Or, ces derniers temps, G. M. Kohls, que je tiens à remercier ici, a bien voulu me signaler la ressemblance, qu'il constatait, de cette nymphe avec celle de $I x$. petauristae Warburton, 1933. Il a même poussé son amabilité jusqu’à me faire parvenir des spécimens de larves et de nymphes de $I x$. petauristae pour me permettre la comparaison.

Il ressort de cette comparaison que, tant par la morphologie générale que par la mensuration des pièces chitineuses, ces deux nymphes peuvent être considérées comme appartenant à la même espèce.

Le tableau ci-dessous où toutes les mensurations sont exprimées en $\mu$ en donnera une idée.

\begin{tabular}{|c|c|c|}
\hline & petauristae & pseudo-holocyclus \\
\hline Longueur totale $\ldots \ldots \ldots \ldots$ & 1.970 & 2.630 \\
\hline $\begin{array}{l}\text { De l'apex au b. postéro-sup. du } \\
\quad \text { rostre } \ldots \ldots \ldots \ldots\end{array}$ & 673 & 813 \\
\hline $\begin{array}{l}\text { Portion denticulée de l'hypos- } \\
\text { tom } \ldots \ldots \ldots \ldots \ldots \ldots \ldots\end{array}$ & 391 & 326 \\
\hline Nombre de dents $\ldots \ldots \ldots \ldots$ & $10-12$ & 13 \\
\hline $\begin{array}{l}\text { Sella : } \\
\quad \text { longueur } \ldots \ldots \ldots \ldots \ldots \ldots \\
\text { largeur }\end{array}$ & $\begin{array}{l}252 \\
112\end{array}$ & $\begin{array}{l}257 \\
102\end{array}$ \\
\hline $\begin{array}{l}\text { Largeur maxima de la base du } \\
\text { rostre } \ldots \ldots \ldots \ldots \ldots \ldots \ldots\end{array}$ & 352 & 400 \\
\hline Ecusson : & & \\
\hline longueur $\ldots \ldots \ldots \ldots \ldots$ & 855 & 1.022 \\
\hline largeur $\ldots \ldots \ldots \ldots \ldots$ & 680 & 760 \\
\hline Palpe: longueur $\ldots \ldots \ldots \ldots$ & 445 & 725 \\
\hline
\end{tabular}

En conclusion, si l'on veut à tout prix trouver une différence entre ces deux tiques, on peut considérer Ix. holocyclus comme une forme «magna» de Ix. petauristaz. 